Since $1964^{5}$ a number of trials of anticoagulant therapy have been carried out in which treated and untreated patients were divided into two groups by random selection, but many of these have not been "double blind." "-8 Such "double blind" studies as have been carried out have not been in complete agreement. G. Aspenström and K. KorsanBengsten ${ }^{9}$ concluded that there was no difference in mortality between patients treated with long-term anticoagulants and untreated controls. C. Merskey and A. Drapkin in their preliminary report came to a similar conclusion, ${ }^{10}$ though their study is not yet complete. E. A. Loeliger and colleagues found no difference in mortality but a higher rate of reinfarction in the untreated patient, ${ }^{11}$ while O. J. A. T. Meuwissen and colleagues found significant benefit from anticoagulant therapy in their patients. ${ }^{12}$ An international review group has recently analysed the records of 2,205 males and 282 females included in nine controlled trials between 1950 and 1965 and could not reach an agreed decision. The only conclusion that can be drawn from these studies is that if any beneficial effect exists it must be marginal.

In their critical review of the published work up to 1960 , Sir John McMichael and E. H. O. Parry ${ }^{4}$ concluded that the value of long-term anticoagulant therapy was unproved, and this remains true today. They showed that the prospect of surviving for five years after recovery from an acute myocardial infarct was $66 \%$ in untreated patients under the age of 60 , being worse with increasing age and complications and better with improving social circumstances and completeness of recovery. The figure varies from $80 \%$ in the doctors studied by J. N. Morris and his colleagues ${ }^{14}$ to $30 \%$ in elderly patients. ${ }^{15} \mathrm{~A}$ reduction in mortality must be the main criterion for the success of anticoagulant treatment. Though the recurrence rate of infarction is also of importance, this is much more difficult to assess. In some of the reports recording favourable effects of anticoagulants patients in the control series did not survive as long as the established mean for untreated patients. Conversely it has been pointed out that

1 Conrad, L. L., Kyriacopoulos, J. D., Wiggins, C. W., and Honick, G. L., Archives of Internal Medicine, 1964, 114, 348.

Armitage, P., and Borchgrevink, C. F., Archives of Internal Medicine, $1966,270,118$

${ }^{3}$ Report of the Working Party on Anticoagulant Therapy in Coronary Thrombosis to the Medical Research Council, British Medical fournal, 1959, 2, 803 ; 1964, 2, 837.

McMichael, J., and Parry, E.H.O., Lancet, 1960, 2, 991

British Medical fournal, 1964, 2, 831

Co-operative Study, fournal of the American Medical Association, 1965, 193, 929.

Ebert, R. V., et al., fournal of the American Medical Association, $1969,207,2263$.

Lovell, R. R. H., Denborough, M. A., Nestel, P. J., and Goble, A. J., Medical fournal of Australia, 1967, 2, 97.

- Aspenström, G., and Korsan-Bengsten, K., Acta Medica Scandinavica, 1966, 176, 563.

- Merskey, C., and Drapkin, A., Blood, 1965, 25, 567.

Loeliger, E. A., et al., Acta Medica Scandinavica, 1967, 182, 549.

2 Meuwissen, O. J. A.' T., Vervoorn, A. C., Cohen, O., and Jordan, F. L. J., Thrombosis et Diathesis Haemorrhagica, 1966, Supplemen No. $21,329$.

3 International Anticoagulant Review Group, Lancet, 1970, 1, 203.

Morris, J. N., Heady, J. A., and Barley, R. G., British Medical fournal, 1952, 1, 503.

5 Eckerström, S., Acta Medica Scandinavica, 1951, 139, Supplement No. 250.

16 Mustard, J., in Anticoagulants and Fibrinolysins, p. 433, ed. R. MacMillan and J. Mustard. Philadelphia, Lea and Febiger. 1961.

Gilchrist, A. R., and Tulloch, J. A., Scottish Medical fournal, 1956, Gilchrist,

18 Paterson, J. C., Mills, J., and Moffatt, T., Archives of Pathology, $1957,64,129$

19 Seaman, A. J., Griswold, H. E. Reaume, R. B., and Ritzmann, L. New England fournal of Medicine, 1969, 281, 115.

20 Pollard, J. W., Hamilton, M. J., Christensen, N. A., and Achor, R. W. P., Circulation, 1962, 25, 311.

21 Pastor, B. 'H., Resnick, M. E., and Rodman, T., fournal of the American Medical Association, 1962, 180, 747.

22 Bjerkelund, C. J., Acta Medica Scandinavica, 1957, 158, Supplement No. 330.

23 Marshall, J., and Shaw, D. A., Lancet, 1960, 1, 995.

24 Singer, A., and Rob, C., British Medical fournal, 1960, 2, 633. mortality figures in some series of patients treated with anticoagulants are less than a life insurance company would expect for a healthy population of the same age and sex. ${ }^{16}$

In other studies the action of anticoagulants is said to be favourable only for the early years of treatment. ${ }^{7}$ If the action of anticoagulants is to prevent arterial thrombosis there is no reason why this prophylactic action should cease. It has been shown too that mural thrombi over infarcts occur with equal frequency in patients on anticoagulants as in those not receiving any specific treatment. ${ }^{17}$ At the same time it must be remembered that recurrent thrombosis is responsible for only little more than half the deaths following myocardial infarction. ${ }^{4}$ Other factors include the thickening of atheromatous plaques and subintimal haemorrhage. ${ }^{18}$ Falls in blood pressure may also be responsible by reducing perfusion through critically narrowed vessels. ${ }^{4}$

The disillusionment in long-term anticoagulant prophylaxis receives further support in a recent report by A. J. Seaman and his colleagues. ${ }^{19}$ This was a double-blind study of 256 patients over an average period of six years, and it showed that long-term prophylactic anticoagulant therapy after acute infarction did not reduce either the mortality or the recurrence rate of infarction. In fact, more of the treated patients required admission to hospital. It is agreed that long-term anticoagulants are of no benefit to women. Any benefit to men is unproved. The physician must therefore weigh these dubious advantages against the potential risks of his treatment.

Most of the haemorrhagic complications occur while the patient's prothrombin levels are in the desired therapeutic range. ${ }^{20}$ The hazards of anticoagulant therapy are significant even in the most experienced hands. The reported incidence of bleeding among patients on anticoagulant therapy is up to $40 \%$ in those who are ambulant, though it is only serious in $2 \%$ to $10 \%$ of patients..$^{12}$ In the series reported by C. J. Bjerkelund ${ }^{22}$ lethal haemorrhage occurred in 4 out of 118 patients on anticoagulants.

While anticoagulants are of value in the treatment of venous thrombosis, this is not true of arterial thrombosis whether cerebal thrombosis ${ }^{23}$ or occlusive arterial disease of the legs. ${ }^{24}$ The inhernt dangers of this form of treatment together with the absence of any evidence of benefit indicates that the time has come to abandon the long-term use of anticoagulants after myocardial infarction.

\section{Holiday Typhoid and T.A.B.}

As a result of hygienic measures, vaccination, and chemotherapy enteric fevers are nowadays less common and less severe than they used to be. But they can still be a menace to travellers, especially to holidaymakers, who are apt to take too few, if any, precautions against them. ${ }^{1}$

In the last decade the World Health Organization has conducted a series of controlled field trials in four countries where enteric infections are endemic. ${ }^{2-5}$ These trials have proved at least two things. Firstly, it is possible by means of a monovalent typhoid vaccine to protect people to a large extent against typhoid fever. Secondly, no laboratory method of assaying a vaccine gives results that can be correlated with those of a controlled field trial in which incidence of typhoid is compared in vaccinated and unvaccinated persons. Paratyphoid fever is 
in a different category. No controlled trial with a paratyphoid A vaccine has yet been reported, and in at least two trials paratyphoid B vaccine has been ineffective. ${ }^{6}$

It has been suggested that a monovalent vaccine would be preferable to a multivalent. W.H.O. experts affirm that it does not seem advisable to add to typhoid antigen (which is potent and effective) an antigen of doubtful effectiveness that adds to the reaction in the vaccinated person but does not necessarily add to the protection against typhoid fever. ${ }^{7}$ In the U.S.A. also there is a trend to change from T.A.B. to a typhoid vaccine ${ }^{8}$ paratyphoid vaccines are thought there to be ineffective and to increase the incidence of reactions. ${ }^{9}$ This is perhaps a challenge to the pharmaceutical industry in the United Kingdom to make a potent monovalent vaccine. An acetoneinactivated and acetone-dried vaccine is marginally more effective than the traditional heat-killed and phenolized preparation, but this might or might not be commercially feasible. All things considered, it does not at present seem essential to discard our time-honoured and successful T.A.B. vaccine.

As to dosage, some have suggested that three doses are unnecessary and that one dose is sufficient to give protection. This may be wishful thinking. The reason why, for example, one dose of a potent typhoid vaccine was as effective as two doses in Guyanese children ${ }^{10}$ was probably that the inhabitants of that area, where typhoid is endemic, had previously ingested subinfective doses of typhoid and related organisms, so that the vaccine was actually a secondary stimulus to a latent immunity. There can be no justification for assuming that in Britain there is any basal immunity to enteric infections. Two doses of T.A.B. vaccine are therefore necessary for protection, and three doses are even better.

The reaction to T.A.B. inoculation is sometimes troublesome, and any means of reducing it is welcome. Probably the most successful method is to give the vaccine intradermally. ${ }^{11} 12$ It is generally agreed that an intradermal dose of $0.1 \mathrm{ml}$. is as effective as a subcutaneous or intramuscular dose of $0.5 \mathrm{ml}$. The British Army has for many years given its T.A.B. (usually combined with tetanus vaccine as T.A.B.T.) intradermally with great success. ${ }^{13}$

What advice, then, should be given on T.A.B. inoculations to travellers going abroad on holiday? Those who have never had any anti-typhoid immunization should have the full course of three inoculations-two doses before travelling, with insistence that the third dose is necessary in 6-12 months' time. Persons who have had the full course three years or more previously should be given two doses of T.A.B. vaccine, with an interval between them of 4-6 weeks. The traveller who leaves his immunization until a week or two before departure should be given one dose of T.A.B.-not two doses

1 British Medical fournal, 1969, 3, 605.

2 Yugoslav Typhoid Commission, Bulletin of the World Health Organization, 1964, 30, 623.

3 Typhoid Panel, U.K. Department of Technical Co-operation, Bulletin of the World Health Organization, 1964, 30, 631.

4 Hejfec, L. B., Bulletin of the World Health Organization, 1965, 32, 1.

Polish Typhoid Committee, Bulletin of the World Health Organization, 1966, 34, 211 .

6 Standfast, A. F. B., British Medical Bulletin, 1969, 25, 189.

7 Cvjetanovic, B., and Uemura, K., Bulletin of the World Health Organization, 1965, 32, 29.

8 U.S. Department of Health, Education and Welfare; Advisory Committee on Immunization Practiçes, Morbidity and Mortality Weekly Report, 1966, 15, 247.

- Kline, S. A., Fournal of Occupational Medicine, 1968, 10, 285.

10 Ashcroft, M. T., et al., Lancet, 1967, 2, 1056.

1 Barr, M., Sayers, M. H. P., and Stamm, W. P., Lancet, 1959, 1, 816.

12 Noble, J. E., Fournal of the Royal Army Medical Corps, 1963, 109, 178.

13 Ministry of Defence, Memorandum on Immunological Procedures, 4th ed., London, H.M.S.O. 1968.

14 Medical Officer, 1970, 123, 12 within 10 days-and reminded that he should have the second dose on his return from holiday. Those who have had the full course of three inoculations within the previous three years require only one reinforcing dose of T.A.B. vaccine.

For the reinforcement of immunity it is logical to assume that, after primary immunization or after a reinforcing dose of vaccine, protection is maintained for at least one year and basal immunity exists for three years. Persons at risk (as holiday travellers generally are) require a reinforcing dose ever year, while those not at risk (persons remaining in this country) should receive this only every third year. It is advisable for people who only occasionally go to places where they may be exposed to typhoid infection to keep their basal immunity "topped up" in order to circumvent the necessity, when they do decide to travel, of undertaking primary immunization afresh. Likewise, the traveller who pops over to the Costa Brava every summer needs only one dose of T.A.B. each time he buys his ticket-three or four weeks before departure.

It is good to know that the Ministry of Health and Social Security is planning to issue information to travel agents and others associated with the tourist trade so that they can pass on the appropriate advice to travellers. ${ }^{14}$

\section{Surgical Treatment of Thyrotoxicosis}

Many clinicians regard subtotal thyroidectomy as the best treatment for hyperthyroidism in young adults with enlarged thyroid glands; but in different series ${ }^{1-5}$ the incidence of hypothyroidism after operation has ranged from 5 to $36 \%$ and of hyperthyroidism from 0 to $11 \%$.

The report by Dr. A. J. Hedley and his colleagues in this week's $B . M . \mathcal{F}$. (page 519) is a useful addition to our knowledge on this subject. They surveyed 254 patients treated by subtotal thyroidectomy in Aberdeen from 1946 to 1965 . Of these only $198(78 \%)$ could be identified despite a thorough search, so that the total morbidity in the series could not be accurately assessed. Data are presented on 146 of these patients operated on in the same surgical unit and followed up for $2-21$ years (mean 9.3 years) after operation. At the time of follow-up $55 \%$ of the patients were euthyroid, $5 \%$ had equivocal thyroid status, $27 \%$ had become hypothyroid and were receiving some form of replacement therapy, $10 \%$ had untreated hypothyroidism, and $4 \%$ were hyperthyroid. Thus

1 Bowers, R. F., American Surgeon, 1966, 32, 89.

Plested, W. G., and Pollock, W. F., Archives of Surgery, 1967, 94, 517.

3 Roy, A. D., Allan, J., and Harden, R. M., Lancet, 1967, 2, 684

4 McNeill, A. D., and Thomson, J. A., British Medical fournal, 1968, 3, 643. Murley, R. S., and Rigg, B. M., British fournal of Surgery, 1968, 55, 757.

Bronsky, D., Kiamko, R. T., and Waldstein, S. S., Archives of Internal Medicine, 1968, 121, 113.

8 Whitsell, F. B., and Black, B. M., fournal of Clinical Endocrinology, 1949, 9, 1202 .

9 Greene, $\mathrm{R}$., Fournal of Endocrinology, 1951, 7, 1.

10 Irvine, W. J., and Stewart, A. G., in Thyrotoxicosis, Proceedings of an International Symposium, Edinburgh, 1967, ed.W. J. Irvine, p. 111. Edinburgh, Livingstone.

11 McLarty, D. G., Alexander, W. D., Harden, R. M., and Clark, D. H., British Medical fournal, 1969, 3, 203. 